\title{
OF-FL: QoS-Aware Fuzzy Logic Objective Function for the RPL Routing Protocol
}

\author{
Olfa Gaddour*, Anis Koubâa ${ }^{\dagger}$, Nouha Baccour ${ }^{\mathbb{I}}$, Mohamed Abid* \\ * CES Laboratory, National School of Engineers of Sfax (University of Sfax), Tunisia \\ $\dagger$ Prince Sultan University, Riyadh, Saudi Arabia \\ $\S$ CISTER/INESC-TEC, ISEP, Polytechnic Institute of Porto, Porto, Portugal \\ II REDCAD Research Unit, National school of Engineers of Sfax(University of Sfax), Tunisia \\ Emails: olfa.gaddour@enis.rnu.tn, aska@isep.ipp.pt, nabr@isep.ipp.pt, mohamed.abid@enis.rnu.tn
}

\begin{abstract}
Low power and lossy networks (LLNs) require efficient routing protocols that should meet the requirements of the critical applications, such as real-time, reliability and high availability. RPL has been recently proposed by the ROLL working group as a tree routing protocol specifically designed for LLNs. It relies on objective functions to construct routes that optimize or constrain a routing metric on the paths. However, the working group did not specify the set of metrics and/or constraints to be used to specify the preferred path, and left it open to implementations. In this paper, we design $O F-F L$, a novel objective function that combines a set of metrics in order to provide a configurable routing decision based on the fuzzy parameters. OF-FL has the advantage to consider the application requirements in order to select the best paths to the destination. Our evaluation with a large-scale testbed in ContikiOS reveals that OF-FL can achieve remarkable performance of the RPLbased LLNs in comparison with the existing objective functions, and appropriately satisfy the quality of service contract of the different applications.
\end{abstract}

\section{INTRODUCTION}

In the last few years, routing in LLNs is considered as one of the key issues that are worth investigation. LLNs stand for networks with very limited resources in terms of energy, computation and bandwidth turning them highly exposed to packet losses. RPL (IPv6 Routing protocol for LLNs), which is the main candidate to act as the standard routing protocol for IPv6 based LLNs such as wireless sensor networks (WSNs), has been recently promoted from an IETF draft to an Internet request for comments (RFC) [1], and thus, it is gaining a lot of maturity. Although RPL has been recently released, several research works have been devoted to investigate the issues that are left open by the working group. However, their goals were to propose some broadcast and multicast mechanisms [2-4], some security countermeasures $[5,6]$ or mobility mechanisms $[7,8]$ and did not consider the application requirements in their proposals.

In this paper, we are interested in improving the quality of service contract of various application areas of LLNs. In the RPL specification, the component that is responsible of selecting paths is called Objective Function (OF). The OF allows to select the preferred parent among the set of neighbors to be the next hop to the DAG root. The RPL specification did not impose any constraint in selecting the routing metrics to consider when choosing the best paths to the root, and thus offers a great flexibility for supporting various application requirements.

There is a wide scope of application areas for LLNs, including industrial monitoring [9], urban sensor networks [10] and building automation [11]. As RPL is intended to be the standard routing protocol for LLNs, which includes all the application requirements enumerated in [9][10] [11], it is necessary to tailor the routing protocol with these requirements.

Several Routing metrics and constraints have been recommended to be used for LLNs. They are classified as either node or link metrics. In addition, existing objective functions use to optimize a unique routing criteria to choose the best path. However, applications of LLNs are varied and may have different and antagonistic requirements. In order to better choose the best path to the root while meeting the application requirements, we propose to design a new objective function that combines several important routing metrics. Fuzzy logic [12] is a well-known model with a powerful theoretical background that combines approximate values of any range. Thus in this paper, we resort to fuzzy logic in order to compute the best path to the root by considering several parameters. We have selected four routing metrics that are stated in linguistic terms and combined with a set of fuzzy rules, and the evaluation of these rules gives the membership of the neighbor node to the fuzzy subset of parents having good quality.

The remainder of this paper is organized as follows: in section 2, we give a brief overview on the RPL routing protocol. In section 3, we provide a literature review of relevant and recent works around the proposed objective functions in RPL. We introduce our fuzzy logic objective function (OF-FL) in section 4. Details of simulation scenarios and results are given in section 5. Finally, Section 6 concludes the paper and discusses future work.

\section{RPL OVERVIEW}

RPL is a distance-vector and a source routing protocol that is designed to operate on top of several link layer mechanisms including IEEE 802.15.4 PHY and MAC layers [1]. It mainly 
targets collection-based networks, where nodes periodically send measurements to a collection point. A key feature of RPL is that it represents a specific routing solution for low power and lossy networks. The protocol was designed to be highly adaptive to network conditions and to provide alternate routes, whenever default routes are inaccessible.

RPL is based on the topological concept of Directed Acyclic Graphs (DAGs). The DAG defines a tree-like structure that specifies the default routes between nodes in the LLN. However, a DAG structure is more than a typical tree in the sense that a node might associate to multiple parent nodes in the DAG, in contrast to classical trees where only one parent is allowed. More specifically, RPL organizes nodes as Destination-Oriented DAGs (DODAGs), where most popular destination nodes (i.e. sinks) or those providing a default route to the Internet (i.e. gateways) act as the roots of the DAGs.

In the construction process of the network topology, each router identifies a stable set of parents on a path towards the DODAG root, and associates itself to a preferred parent, which is selected based on the Objective Function. The Objective Function defines how RPL nodes translate one or more metrics into ranks, and how to select and optimize routes in a DODAG. It is responsible for rank computation based on specific routing metrics (e.g. delay, link quality, connectivity, etc. ...) and specifying routing constraints and optimization objectives [13].

\section{RELATED WORK}

Several routing metrics have been recommended to be used for LLNs [14-17]. However, the specification of RPL did not impose any routing metric and left it open to implementations. The proposed objective functions by the IETF presented in [14] and [15] have defined some recommendations on how to implement the OFs without specifying the routing metrics that can be used.

In [15], the default objective function for RPL, referred to as OF0, was first to be proposed. OF0 is a simple $\mathrm{OF}$, where the node always chooses the neighbor with the minimum rank as the preferred parent. The rank is defined as an integer representing the node's individual location within the DODAG; it strictly decreases in the downlink direction from the DAG root to the RPL routers. Thus, this OF does not consider any routing metric specified in [18]. OF0 is designed to be the common OF that will allow inter-operation between the different implementations of RPL. With this OF, all the upward traffic is routed via the preferred parent with no attempt to perform any load balancing.

In [14], MRHOF was proposed. Unlike OF0, MRHOF is based on metric containers, which is a common header for metrics and constraints objects located in the DIO control message. The metric container is used with several flags to specify the nature and features of routing objects, such as whether it represents a represents a metric or a constraint, it is local or global, additive or multiplicative, etc. In MRHOF, the path cost represents the cost in terms of the selected metric, from a node to the root through the neighbor. A node computes the path cost by adding two components $(i)$ The selected metric for the link to a candidate neighbor (ii)the value of the selected metric in the DIO sent by that neighbor. MRHOF has been implemented with a single metric with the link ETX metric [19] [20], the node remaining energy metric [16] [20] and the delay link metric [17]. In addition, the ETX has been widely used in the recent research papers [21] [8] [22].

In [23], the authors proposed the combination of two routing metrics among the following (i.) the hop count, (ii.) the ETX, (iii.) the remaining energy and (iv.) the RSSI in RPL routing decision process. They proposed two methods to combine the different routing metrics which are simple combination and lexical combination. In simple combination, the rank of the node is evaluated based on a composition function and advertised through the DIO message. The composition function is the sum of the ranks of the two selected metrics with their relative weights that enable the shift of emphasis of the two metrics. In lexical metric composition, the node selects the neighbor with the lower (or greater respectively) value of the first selected metric, and if the first values are equal, the node selects the one with the lower (or greater respectively) value of the second composition metric. Lexical combination leads to performance metric prioritization. However, the ordering of metrics will drive the parent selection without combination of these metrics and can lead to a more costly choice.

In [24], the authors analyzed the impact of the objective functions on the network topology. They compared two objective functions: OF0 and LLQ OF which is based on the link reliability metric RSSI. They measured the OF impact by using their own simulation environment. The paper emphasized the impossibility of selecting one objective function between $\mathrm{OF} 0$ and LLQ OF as the two OFs cause performance deterioration, and concluded that further studies should be done in order to improve the network topology and performance.

In [25], two MAC-based routing metrics were proposed. The first metric considers the ETX and the packet losses due to the MAC contention. The second metric selects the parent that has the lightest traffic load by considering the power consumption and the application required reliability. The authors implemented the proposed metrics in a real testbed composed of seven Telosb motes and measured the end-to-end reliability and the power consumption. However, the authors compared the proposed routing metrics with the backpressure protocol [26] which is not designed for RPL.

\section{A. Limitation of Existing Objective Functions}

As discussed in the previous section, existing objective functions rely either on a single metric or on the combination of two metrics in order to optimize the paths to the DAG root.

The use of a single routing metric in the OF encompasses some advantages and limitations. First, it can be noted that relying on a single metric in an objective function may be inefficient and could degrade the performance of the DAG as it may not fully satisfy the application requirements. For example, while the hop count routing metric allows to choose the shortest path, it may lead to the failure of one or more nodes due to battery depletion, as the battery level is not 
considered in the decision process. In addition, considering ETX as a single routing metric may lead to high latency in routing messages. In fact, while selecting parents with low ETX make the network more reliable, it cannot reduce the latency in routing the messages. Thus, the ETX metric alone is not adequate for real time applications, as it does not consider the timing requirements of the applications.

Some recent works came up with some solutions to overcome the single metric problem. However, combining two metrics is insufficient to efficiently satisfy all the application requirements as the objective of each LLN application may differ from an application to another. In addition, considering two routing metrics may improve the performance in the DAG, but at the cost of the degradation of other performance parameters. For instance, choosing the latency and the ETX metrics may help the RPL routers to use more reliable paths with a minimal latency to reach the DAG root, but may lead to the overuse of some RPL routers leading to battery depletion.

As a conclusion, single routing metric or a simple combination of two routing metrics cannot provide a good assessment on the quality of the routes from the source to the destination in the DAG. As such, there is a need to design a holistic objective function that combines several representative metrics to be able to characterize the route quality in a more efficient way. In order to better choose the best parent as the next hop, we advocate combining several important routing metrics, to get a holistic characterization of the neighbor.

\section{OF-FL: OBJECTIVE FUNCTION FOR RPL BASED ON FUZZY LOGIC}

\section{A. Properties of a Good Route}

In this section, we identify four routing metrics to be considered in the design of OF-FL. Each metric describes an important property of the neighbor node that is eligible of acting as a parent, thus contributing to forming a route towards the root. We consider a subset of link and node metrics that will be used to express the goodness of a given neighbor. First, we identify the properties of a good route. In general, a good route in LLNs shall satisfy the following properties:

- Real-Time: a good route should be able to provide low end-to-end delays, in particular for real-time data flows. The real-time property can be measured through the offered endto-end delay from one source to a destination (i.e. DAG root) through a particular route.

- Reliability: A route is reliable if it provides a high delivery ratio. This property is much correlated with the quality of the links forming the route. Thus, the reliability property can be assessed through link quality estimators, such as the packet reception ratio (PRR), the received signal strength (RSS), the expected number of retransmissions (ETX) and others [27].

- Energy Efficiency: a route is energy efficient if it uses nodes that have more energy than the others. As a consequence, an efficient route selection must consider the battery levels of the nodes to extend the network lifetime. Nodes with low battery levels should be avoided in the routing process as much as possible. The energy-efficiency can be measured through the total consumed energy, or the network lifetime, or the remaining energy, etc.

It is clear that these different properties may have antagonist requirements and it is challenging to satisfy all of them simultaneously. For instance, a reliable route may require longer number of hops thus increasing the end-to-end delay. In [18], several routing metrics have been proposed to be used for routing in LLNs. Our objective is to specify a holistic routing metric that effectively combines individual metrics to jointly consider different requirements in the routing process. We propose a fuzzy logic approach for metric combination in the next section.

\section{B. Metrics of Interest}

As described above, fuzzy logic represents a widely used approach to combine several logical information that are of different nature, using the concept of membership functions [28-30]. The first question that arises when using fuzzy logic is: what are the metrics to be combined ?

In what follows, we consider four representative routing metrics for parent selection, namely:

- End-to-End Delay : This metric represents the sum of link latency, which is defined in [18] as an aggregated additive metric. This metric should be minimized for applications that require real-time guarantees.

- Hop Count: This metric refers to the number of hops between the neighbor node (i.e. the candidate parent) and the root. For real-time applications, it is effective to reach destination through the smallest possible number of hops. However, it may happen that some shortest paths experience larger delays and higher energy dissipation due to congestion/overload. Thus, combining this metric with the delay will be more effective for real-time applications by optimizing the selection process of routes.

- Link Quality: This metric is measured by the expected number of retransmissions (ETX) as an indicator of the link quality between the node and its neighbor. A good link should have ETX values close to zero. High ETX values mean that the link is unreliable as the number of retransmissions goes high. This metric should have significant weight for applications with reliability requirements. Other link quality metrics such as Received Signal Strength (RSS) or Link Quality Level (LQL) may also be considered as link quality estimators [27]. With respect to LQL, it is not recommended to consider it as link quality estimator as it maps the link quality to seven values (from 1 to 7 ), which only gives a rough classification of the current link metric.

- Node Energy: this metric represents the remaining battery level in a RPL router. With this metric, it is possible to avoid selecting routers with low energy, and thus will allow to extend the network lifetime. It is essential to consider this metric for applications with energy-efficiency concerns.

Discussion: It is clear that each metric provides an individual perspective of the notion of a good route. In addition, the aforementioned routing metrics have an important impact on 
the network behavior. It is therefore reasonable to think about accounting for all these metrics simultaneously - as much as possible - to optimize the selection process of good routes that can provide the best possible balance among all of them.

Let us consider the following example to understand the importance of metrics combination. First, the combination of the delay and the hop count allows assessing the number of hops from the router node to the DAG root while taking into account its delay. Now, assume that a node whose path to the root has the lowest delay and at the same time the nearest to the DAG root. This situation makes that node the best candidate for the parent among the set of the other neighbors. However, using this parent node in a repetitive way may rapidly deplete its battery level. Consequently, it is also crucial to consider the node energy in the selection of the best parent in order to avoid overusing a node until it vanishes its energy, and thus, extending the node's availability and the network lifetime in general.

\section{Combination of Routing Metrics}

In this section, we propose an improvement of RPL with the use of a holistic objective function based on the combination of the aforementioned metrics using a fuzzy logic approach. We call the objective function as OF-FL. In the proposed objective function, we consider the aforementioned link and node metrics, end-to-end delay, hop count, ETX and battery level in the design of our objective function. Each metric describes an important link or node property, as previously discussed. The set of selected link and node metrics will be used to assess the quality and compare the set of neighbor nodes to select the best parent that will represent the next hop to reach the DAG root. In order to assess the best neighbor to be the preferred parent, we resort to fuzzy logic.

The use of artificial intelligence techniques to support the decision making process is in fact widely used in the recent research works in relation with low power and lossy wireless sensor networks. Artificial intelligence techniques reinforce the efficiency and performance of routing protocols, by combining data from nodes and their interactions in order to make decision to improve the global network performance. Since we aim to decide about the best parent among the list of neighbors depending on the application requirements in an efficient way, our proposal makes use of fuzzy logic as it plays an important role in decision making. There are many advantages of fuzzy logic:

- It allows an abstract reasoning on values of any range;

- It provides a rigorous algebra for dealing with imprecise information;

- It is a convenient method of combining conflicting objectives and expert human knowledge;

- It can be implemented with low complexity algorithms. In what follow, we detail the main components of the proposed objective function.

1) Linguistic Variables: A key concept in Fuzzy Logic is that a variable could belong to a set that is between true and false. Linguistic variables are input or output variables whose values are words or sentences instead of numerical values. For instance, the linguistic variable of the hop count is a member of three sets according to its position to the DAG: "near", "vicinity" and "far". These sets are called linguistic variables. As shown in Figure 1b, for values of hop count below 2, the membership to the fuzzy subset of nodes that are "near" the DAG is of 1 . Starting from 7 hops, the node is considered totally out of the fuzzy subset of neighbors that are near the root. For values of hop count between 2 and 7, the membership decreases linearly from 1 to 0 . The same reasoning holds for "vicinity" and "far" hop count. In same manner, we classified the ETX, the end-to-end delay and the battery level into three linguistic variables as shown in Figure 1.

2) Membership Functions: The membership functions are used to quantify the linguistic terms. In Figure 1, the membership functions of battery level, hop count, delay and ETX variables are plotted. We have chosen the trapezoidal form for the membership functions as it is largely used in fuzzy logic systems [31]. All the metrics are binned into three fuzzylogic semantic levels and thus have computation complexity. The membership is computed by a membership function that relates a value of a discourse of universe to a real value in the interval $[0-1]$.

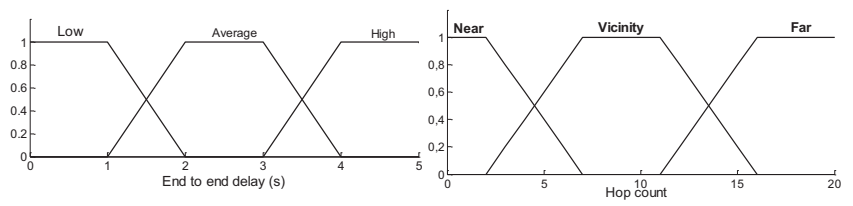

(a) Input variable of the End to end (b) Input variable of the hop count delay
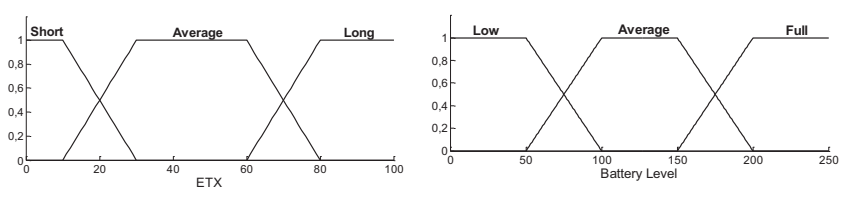

(c) Input variable of the link quality (d) Input variable of the remaining en(ETX) ergy

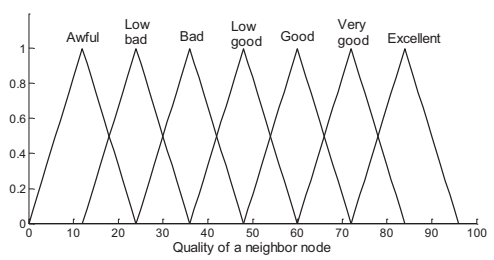

(e) Output variable of neighbor quality

Figure 1: Definition of membership functions

As shown in Figure (1a), the end-to-end delay is a member of three sets which are "low", "average" and "high". Following several simulations, we concluded that the end-to-end delay of a path is in the range between 0 and 15 seconds. We have chosen reasonable values for the thresholds, and we considered 
for instance that when the end-to-end delay is less than 3 seconds, the neighbor node fully belongs to the fuzzy subset of nodes with low latency.

The hop count is a member of three sets which are "near", "vicinity" and "far". The maximum hop count mainly depends on the size of the deployment area and the transmission power of the motes [22]. The hop count thresholds presented in Figure (1b) are inspired from our simulation studies of RPL presented in [22] and strongly related to the area of deployment.

The Expected Transmission Count (ETX) membership function is an important routing metric that indicates the reliability of a path. As shown in Figure (1c), the ETX path metric belongs to the range $[0 . .100]$. We concluded this range from a set of simulations performed with different scenarios [22].

The battery level is measured with an integer encoded in 8 bits. Thus, the energy ranges from 0 (no energy) to 255 (full energy). As shown in the figure, the battery is a member of three sets during its life (see Figure (1d). The choice of these thresholds can be tuned according to the application requirements. For instance, for applications with energy efficiency concerns, one can argue that the battery level begins to be full at $70 \%$. This will have an impact on the choice of the best parent giving more weight for the neighbor node that has the highest remaining energy.

3) Fuzzy Rules: In this section, we present the fuzzy rules that govern the combination of the different metrics, which represent the input fuzzy sets, namely the battery level, the end-to-end delay, the hop count and the ETX. OF-FL considers each of the metrics mentioned in the previous section as a different fuzzy variable. As our fuzzy system is a four-input fuzzy controller with three membership functions for each input, our rule base consists of $3^{4}=81$ rules. A few rules are given in Table I. The rules have been formulated according to the criteria described above. In Table I, the last column contains the output fuzzy variable i.e., the quality of the neighbor. The output fuzzy set which is the neighbor quality consists of seven fuzzy sets from "awful" to "excellent". A quality between 0 and 100 is assigned to each neighbor. Figure (1e) shows the quality output metric. Note that we

\begin{tabular}{|c|c|c|c||c|}
\hline Hop Count & End-to-end delay & Battery level & ETX & Quality \\
\hline Near & low & high & short & Excellent \\
\hline Near & low & high & average & very good \\
\hline Near & low & average & short & very good \\
\hline Near & average & high & long & good \\
\hline Vicinity & low & average & average & good \\
\hline Vicinity & average & high & short & low good \\
\hline Vicinity & high & low & average & bad \\
\hline Vicinity & average & average & long & bad \\
\hline Far & high & high & average & low bad \\
\hline Far & high & average & short & low bad \\
\hline Far & average & low & average & awful \\
\hline Far & high & low & long & awful \\
\hline
\end{tabular}

Table I: Fuzzy rule base

give the best quality to the candidate parent that has high battery level, low hop count, low end-to-end delay and low link
ETX. The fuzzy sets and rules, as well as parameters, can be customized depending on the application requirements, node features and network capabilities. To evaluate the fuzzy rules and the combination of the results of the individual rules, we used the Mamdani implication [12] to represent the meaning of the "if-then" rules.

\section{Defuzzification}

Once all the rules are established, the next step is the defuzzification, which consists in producing one output metric value from several membership values to produce a crisp control action. We have chosen a simple and frequently used defuzzification method which is the centroid defuzzification method [32] that finds the balance point of the solution fuzzy region by calculating the weighted mean of the fuzzy region. Mathematically, the crisp output domain value $\mathrm{R}$, from the solution fuzzy region $\mathrm{A}$, is given by:

$$
R=\frac{\sum_{i=1}^{N} W_{i} \times \mu_{A}\left(W_{i}\right)}{\sum_{i=1}^{N} \mu_{A}\left(W_{i}\right)}
$$

Where $W_{i}$ is the domain value corresponding to the rule $i, \mathrm{~N}$ is the number of rules triggered in the inference engine, and $\mu_{A}\left(W_{i}\right)$ is the predicate truth of that domain value.

\section{Performance Evaluation}

\section{A. Simulation Settings and Objectives}

The objective of this simulation study is to demonstrate how OF-FL can provide a better performance with respect to the application requirements in a RPL-based LLN as compared to the objective functions OF0 and MRHOF. We simulated OFFL under COOJA, a well-known emulator available under Contiki operating system [33]. In all the simulations, we consider the default simulation testbed which consists in a large scale DAG containing up to 100 RPL routers and one DAG root spanning over a squre space $(600 \mathrm{~m}$ by $600 \mathrm{~m})$. The nodes are spread randomly in the space so that they form a connected network. The radio channel is set to 26 and the transmission power to $0 \mathrm{dBm}$, which is the maximum available radio output power level for the simulated nodes (Tmote Sky). In each simulation, each RPL router periodically sends one data packet to the root each 60 seconds. The nodes begin the transmission of data packets after 60 seconds to enable the topology establishment. We run each simulation for a sufficient time (60 minutes) to ensure that simulations converge to a steady state. Each result is measured with a $90 \%$ of confidence interval.

\section{B. Performance Metrics}

The performance metrics analyzed for the evaluation of OFFL are the following:

- Average hop count: It is the average number of hops between each node and the DAG root.

- End-to-end delay: It is the duration between starting packet transmission and its reception by the DAG root. 
- Packet loss ratio: It is the ratio of the total number of delivered packets (at the DAG root) to the total number of sent packets (by all the router nodes): This metric is an indicator of the end-to-end reliability of the routing protocols.

- Average remaining energy: this metric is important to estimate the network lifetime. It is the average battery level remaining in all nodes.

- Average number of parent changes: It is the number of times a node has changed its parent. This metric is an indicator of the topology stability. Too many parent changes lead to unstable topology, but improve the quality of routes and the routing performance (e.g., PDR and RTX) [34].

\section{Simulation Results}

In this section, we evaluate the performance of OF-FL and compare it against the performance of the two other objective functions, namely $\mathrm{OFO}$ and MRHOF.

1) Average Hop Count: Figure 2 compares the average hop count of OF-FL, MRHOF with ETX and OF0. It is clear that the three networks are almost identical in the case of a sparse network composed of less than 50 nodes. This can be justified by the relatively limited number of neighbors which makes the choice of another parent very restricted. However, it is clear that OF-FL distinctly allows a lower average hop count, as compared with MRHOF with ETX, in case of dense networks composed of more than $50 \mathrm{RPL}$ routers. It is obvious that the average hop count of OF-FL becomes closer to that of OF0 under a high node density (more than 80 nodes). This confirms the tendency of OF-FL to minimize the number of hops within the DAG.

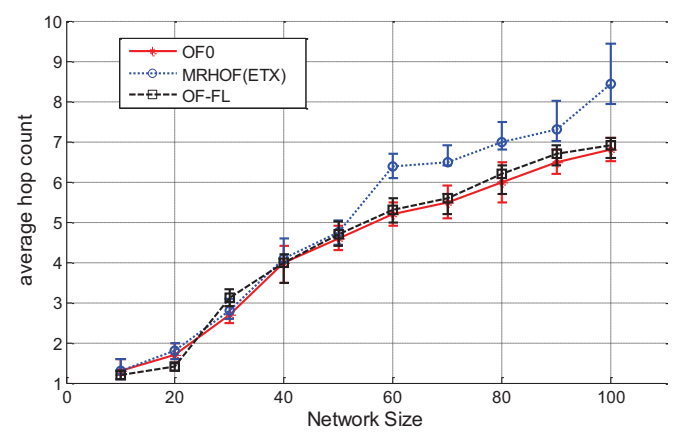

Figure 2: Comparison between OF0, MRHOF with ETX and OF-FL in term of the average hop count

2) Number of Parent Changes: To record the network stability, we have measured the average number of parent changes of OF-FL and compared it with that of OF0 and MRHOF with ETX metric. Figure 3 depicts the number of parent changes of the three objective functions. The first observation is that RPL based networks with OF0 and MRHOF with ETX experienced an average number of 0.2 and 0.252 respectively for each node every hour, which is considered as low. This result is expected as these OFs aim only to minimize the rank without considering any optimization in the process of parent selection. We note also that the OF-FL based network experienced a

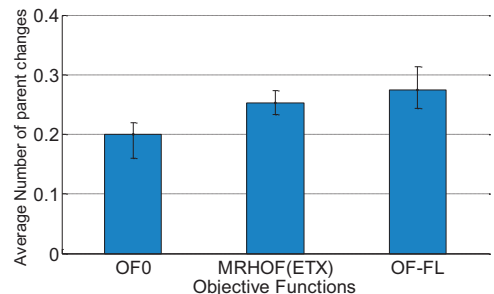

Figure 3: Comparison between OF0, MRHOF with ETX and OF-FL in term of the average number of parent changes

slightly higher number of parent changes per node per hour, which is equal to 0.275 . This relatively high number of parent changes is an indicator of topology instability but has the advantage to improve the quality of routes and the routing performance as we will explain in the next sections.

3) End to End Delay: Figure 4 shows the average end to end delay of the three objective functions OF0, MRHOF with path ETX and OF-FL based on the number of hops to the DAG root. The delay is measured as the time between sending a frame and the reception of its acknowledgement. According to

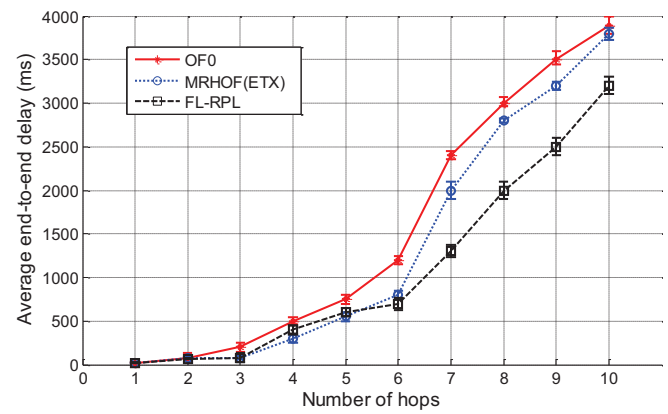

Figure 4: Average End to End Delay of OF0, MRHOF with ETX and OF-FL as a function of the number of hops

the figure, the three objective functions keep an average endto-end delay under 4 seconds. However, OF0 based network induces more latency than the two other schemes, although it allows to minimize the hop count. This result demonstrates that the shortest path does not mean the path that induces less latency, as some nodes may be congested in the shortest path. In addition, the figure shows that OF-FL has a low average end-to-end delay as compared to OF0 and MRHOF with ETX mainly when the distance from the root becomes significant (beginning from 6 hops) and the maximum delay that can undergo a data packet is 3.4 seconds for 10 hops versus 4 seconds for OF0. This result is mainly due to the average hop count that is minimized, and the end to end delay that is considered when deciding about the next hop. These results are promising as OF-FL allows to minimize the end-to-end delay as compared with the existing objective functions.

4) Network Lifetime: In this scenario, we considered a DAG composed of one DAG root and 100 RPL routers. Through our simulations, it was observed that the effect of 
the objective function on the average power consumption is less important. For this reason, we have observed the nodes remaining energy of all the nodes to compare the network lifetime of the RPL routers with the three objective functions and we varied the throughput from 1 to 6 packets per minute. We have run these simulations for 2 simulation days. Figure 5 shows the results of these simulations.

A straightforward observation is that with $\mathrm{OF0}$ and MRHOF with ETX, the remaining energy distribution is not balanced. We note for instance that for OF0 with 1 packet/minute, $23 \%$ of the nodes have a remaining energy under $80 \%, 31 \%$ of the nodes have a remaining energy between $81 \%$ and $85 \%$, $26 \%$ of the nodes between $86 \%$ and $90 \%$, and $10 \%$ of the nodes between $91 \%$ and $92 \%$. This result will have an impact on the network survivability, as some nodes will undergo a completely battery exhaustion early. This observation is emphasized with 6 packets/minute as the nodes will exhaust their batteries much faster. The simulation results also demonstrate that in the case of OF-FL and in both experiments, the energy expenditure is well balanced among the nodes $(86 \%$ of the nodes have a remaining energy between $84 \%$ and $87 \%$, and $14 \%$ of nodes have $90 \%$ of their battery), which is an important property for the network lifetime. It is clear also that in the cases of OF0 and ETX based schemes, there were less-powered nodes when compared to those in the OF-FL based network. Thus, we estimate that OF-FL will delay the battery depletion of the first nodes.

5) Packet Loss Ratio: To measure the reliability of the network with OF-FL, we have compared OF-FL with OF0 and MRHOF in term of packet loss ratio. We also varied the throughput from 1 packet to 6 packets per minute. Figure 6 shows the packet loss ratio as a function of the network size.

From these simulation results, one can see that the packet loss is low in low node density; and this problem becomes more serious under a high node density. We also observe that with a throughput of 1 packet/min, a RPL based network with OF-FL has a much lower packet loss ratio than that with OF0, and almost the same packet loss as an ETX based network. The reason of high packet loss in the OF0 based network is that OF0 does not promote high quality links, and when choosing the parent with minimal rank, this parent may be congested and thus will drop the data packets. An ETX based network has a comparable packet loss ratio as OF-FL, and this result can due to the use of the same link quality estimator which is ETX. Minimizing the ETX when selecting the next hop will imply a path with low packet loss ratio [35]. For the three schemes, we notice that the packet loss ratio is high mainly when the number of hops increases. These results raise the question about the effectiveness of the ETX and it is necessary to promote the reliability by choosing another link quality estimator such as F-LQE [28]. When sensor transmission rates becomes large ( 6 packets per minute), the packet loss ratio increases. This result is mainly due to network congestion and packet collision. We note also that the performance of OF-FL becomes better than MRHOF with ETX as it allows dropping a lower number of data packets. Thus, OF-FL outperforms MRHOF with ETX when the amount of data packets is high, which demonstrates the effectiveness of OFFL in high throughput.

\section{CONCLUSIONS AND Future WORK}

In this paper, we have presented OF-FL, a new objective function for RPL-based LLNs. In comparison with the existing objective functions that rely only on one or two metrics and thus do not consider the applications requirements in the process of path optimization, OF-FL combines four node and link metrics (ETX, hop count, end-to-end delay and battery level) by using Fuzzy Logic, as we proved that this technique is an appropriate strategy for combining different and heterogeneous metrics. The best parent is specified as a set of IF-THEN rules that combines the four linguistic variables. The evaluation of the fuzzy rules returns the membership of the neighbor in the fuzzy subsets of neighbors with good qualities. The neighbor that has the greatest quality will be selected as the preferred parent. OF-FL has been implemented with only minor addons, ensuring backward compatibility with the RPL standard specification. Simulations show that OF-FL has achieved a great improvement in the RPL-based network especially in term of end-to-end delay, network lifetime and packet loss ratio. Thus, our solution intent to support multiple concurrent applications with antagonistic requirements simultaneously.

Future work will address the application of OF-FL in a heterogeneous wireless sensor networks where different applications can be deployed. Our aim is to fine tune the fuzzy parameters designed in OF-FL in order to select the paths that are suitable for each application.

\section{REFERENCES}

[1] T. Winter, P. Thubert, A. Brandt, J. Hui, and R. Kelsey, "RPL: IPv6 Routing Protocol for Low Power and Lossy Networks," RFC 6550, March 2012.

[2] T. Clausen and U. Herberg, "Multipoint to Point and Broadcast in RPL," 13th International Conference on Network-Based Information Systems, pp. $1-6$, September 2010.

[3] T. Clausen and U. Herberg, " Comparative Study of RPL-Enabled Optimized Broadcast in Wireless Sensor Networks," 6th International Conference on Intelligent Sensors, Sensor Networks and Information Processing, Brisbane, Australia, December 2010.

[4] G. C. Oikonomou and I. W. Phillips, "Stateless multicast forwarding with RPL in 6LowPAN sensor networks," in PerCom Workshops, pp. 272-277, 2012.

[5] A. Dvir, T. Holczer, and L. Buttyán, "VeRA - Version Number and Rank Authentication in RPL," 7th IEEE International Workshop on Wireless and Sensor Networks Security, IEEE, 2011.

[6] T. Tsao, R. Alexander, M. Dohler, V. Daza, and A. Lozano, "A Security Framework for Routing over Low Power and Lossy Networks," IETF Requirement Draft, March 2012.

[7] L. B. Saad and B. Tourancheau, "Sinks Mobility Strategy in IPv6-based WSNs for Network Lifetime Improvement," International Conference on New Technologies, Mobility and Security IFIP NTMS, 2011.

[8] K. C. Lee, R. Sudhaakar, L. L. Dai, S. Addepalli, and M. Gerla, "RPL under mobility," in Consumer Communications and Networking Conference, pp. 300-304, 2012.

[9] E. K. Pister, E. P. Thubert, S. Dwars, and T. Phinney, " Industrial Routing Requirements in Low-Power and Lossy Networks," RFC: 5673, March 2009.

[10] E. M. Dohler, E. T. Watteyne, E. T. Winter, and E. D. Barthel, “ Routing Requirements for Urban Low-Power and Lossy Networks," RFC 5548, March 2009.

[11] A. Brandt, J. Buron, and G. Porcu, "Home Automation Routing Requirements in LLNs," RFC 5826, March 2010. 


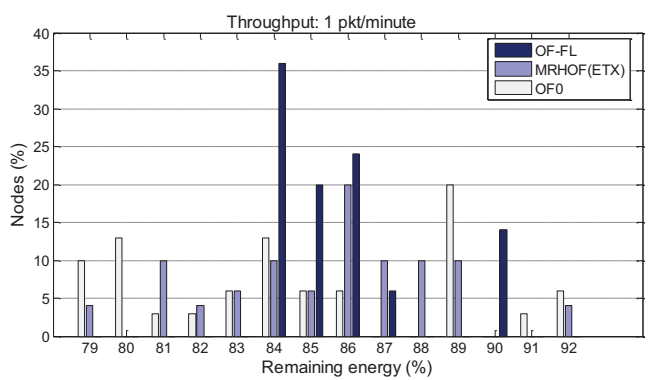

(a) Power distribution with $1 \mathrm{pkt} / \mathrm{minute}$

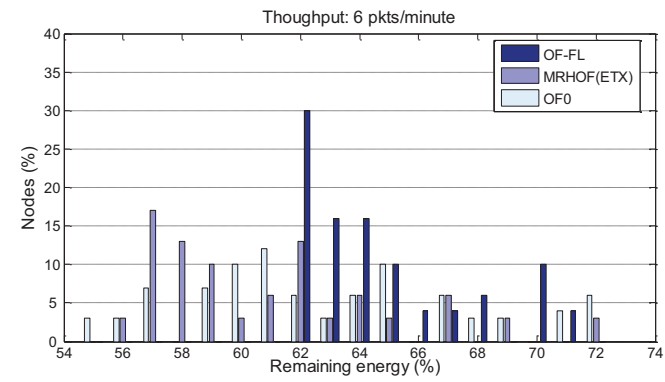

(b) Power distribution with 6 pkts/minute

Figure 5: Remaining power distribution for different packet transmission rates

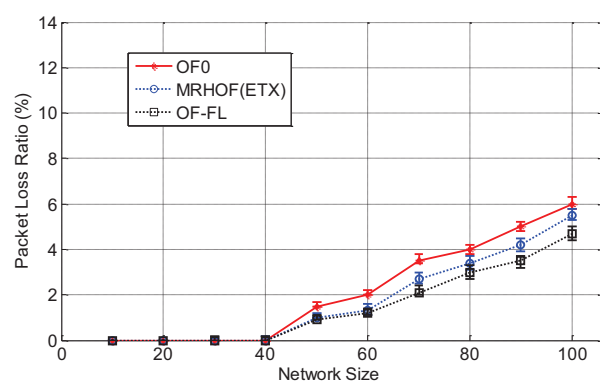

(a) packet loss ratio with $1 \mathrm{pkt} / \mathrm{minute}$

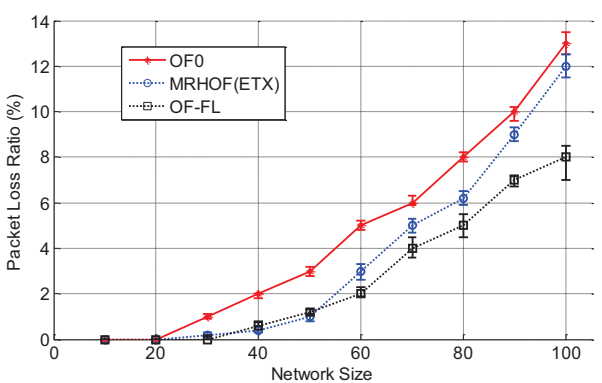

(b) packet loss ratio with 6 pkts/minute

Figure 6: Comparison between OF0, MRHOF with ETX and OF-FL in term of packet loss Ratio

[12] A. S. Mamdani, E. H., "Int. j. man-machine studies," An experiment in linguistic synthesis with a fuzzy logic controller, vol. 7, pp. 1-13, 1975.

[13] O. Gaddour and A. Koubaa, "Survey RPL in a nutshell: A survey," Computer Networks, vol. 56, no. 14, pp. 3163-3178, 2012.

[14] O. Gnawali and P. Levis, "The ETX Objective Function for RPL," IETF Internet Draft: draft-gnawali-roll-etxof-00, 2010.

[15] P. Thubert, "Objective Function Zero," RFC 6552, March 2012.

[16] P. O. Kamgueu, E. Nataf, and T. Djotio, "Energy-based routing metric for RPL," 2013.

[17] P. Gonizzi, R. Monica, and G. Ferrari, "Design and evaluation of a delay-efficient RPL routing metric," in IWCMC, pp. 1573-1577, 2013.

[18] J. Vasseur, M. Kim, K. Pister, N. Dejean, and D. Barthel, "Routing Metrics used for Path Calculation in Low Power and Lossy Networks," RFC 6551, March 2012.

[19] N. Tsiftes, J. Eriksson, and A. Dunkels, " Poster Abstract: Low-Power Wireless IPv6 Routing with ContikiRPL," IPSN, Stockholm, Sweden., pp. 12-16, 2010.

[20] L. Bartolozzi, T. Pecorella, and R. Fantacci, "ns-3 RPL module: IPv6 Routing Protocol for Low power and Lossy Networks," ACM, 62012

[21] L. B. Saad, C. Chauvenet, and B. Tourancheau, "Simulation of the RPL Routing Protocol for IPv6 Sensor Networks," in 5th International Conference on Sensor Technologies and Applications, 2011.

[22] O. Gaddour, A. Koubaa, S. Chaudhry, M. Tezeghdanti, and M. Abid, "Simulation and Performance Evaluation of DAG Construction with RPL," in 3rd International Conference on Communications and Networking (ComNet), 2012.

[23] P. Karkazis, H. C. Leligou, P. Trakadas, L. Sarakis, T. H. Velivassaki, and C. Capsalis, "Design of primary and composite routing metrics for RPL-compliant Wireless Sensor Networks," in Telecommunications and Multimedia (TEMU), 2012.

[24] A. Brachman, "RPL Objective Function Impact on LLNs Topology and Performance," Internet of Things, Smart Spaces, and Next Generation
Networking, vol. 8121, pp. 340-351, 2013.

[25] P. Di Marco, C. Fischione, G. Athanasiou, and P.-V. Mekikis, "MACaware routing metrics for low power and lossy networks," in INFOCOM, Proceedings IEEE, pp. 13-14, 2013.

[26] S. Moeller, A. Sridharan, B. Krishnamachari, and O. Gnawali, "Routing Without Routes: The Backpressure Collection Protocol," in IPSN, pp. 279-290, 2010.

[27] N. Baccour, A. Koubâa, L. Mottola, M. A. Zúñiga, H. Youssef, C. A. Boano, and M. Alves, "Radio link quality estimation in wireless sensor networks: A survey," ACM Trans. Sen. Netw., vol. 8, pp. 34:1-34:33, 2012.

[28] N. Baccour, A. Koubaa, H. Youssef, M. B. Jamâa, D. do Rosário, M. Alves, and L. B. Becker, "F-LQE: A fuzzy link quality estimator for wireless sensor networks," in EWSN, pp. 240-255, 2010.

[29] R. L. Gomesa, W. M. Juniorb, E. Cerqueiraa, , and A. J. Abeléma, Using fuzzy link cost and dynamic choice of link quality metrics to achieve QoS and $Q o E$ in wireless mesh networks. 2010.

[30] A. Ortiz, T. Olivares, J. Castillo, L. Orozco-Barbosa, P. Marron, and F. Royo, "Intelligent role-based routing for dense wireless sensor networks," in Wireless and Mobile Networking Conference (WMNC), 2010 Third Joint IFIP, pp. 1-6, 2010.

[31] J. F. Brule, "Fuzzy systems - a tutorial."

[32] T. Takagi and M. Sugeno, "Fuzzy identification of systems and its applications to modeling and control," Systems, Man and Cybernetics, IEEE Transactions on, vol. SMC-15, pp. 116-132, 1985.

[33] "Contiki Operating System."

[34] N. Baccour, A. Koubaa, M. B. Jamâa, H. Youssef, M. Zuniga, and M. Alves, "A comparative simulation study of link quality estimators in wireless sensor networks," in MASCOTS, pp. 1-10, 2009.

[35] N. Liu and W.-G. Seah, "Performance evaluation of routing metrics for community Wireless Mesh Networks," in ISSNIP, pp. 556-561, 2011. 